\title{
Quenching of Light Hadron Spectra in p-A Collisions from Fully Coherent Energy Loss
}

\author{
François Arleo $\oplus^{1}$ and Stéphane Peigné $\oplus^{2}$ \\ ${ }^{1}$ Laboratoire Leprince-Ringuet, École polytechnique, Institut polytechnique de Paris, CNRS/IN2P3, 91128 Palaiseau, France \\ ${ }^{2}$ SUBATECH UMR 6457 (IMT Atlantique, Université de Nantes, IN2P3/CNRS), 4 rue Alfred Kastler, 44307 Nantes, France
}

(Received 20 March 2020; revised 22 May 2020; accepted 17 June 2020; published 14 July 2020)

\begin{abstract}
We single out the role of fully coherent induced gluon radiation on light hadron production in $p$-A collisions. The effect has an interesting color structure, as the induced radiation depends on the global color charge of the partonic subprocess final state. Baseline predictions for light hadron nuclear suppression in $p$ - $\mathrm{Pb}$ collisions at the LHC are provided, taking into account only the effect of fully coherent energy loss, which proves of the same order of magnitude as gluon shadowing or saturation. This underlines the need to include fully coherent energy loss in phenomenological studies of hadron production in $p$-A collisions.
\end{abstract}

DOI: 10.1103/PhysRevLett.125.032301

The wealth of hadron production data in proton-nucleus collisions at collider (RHIC, LHC) energies allows for a detailed study of parton dynamics in cold nuclear matter. Over the last two decades, several approaches, in particular the color glass condensate [1] or the use of nuclear parton distribution functions (NPDFs) within collinear factorization [2], have been used in phenomenological studies of hadron production in high-energy $p$-A collisions [3-10]. To our knowledge, none of these approaches have yet addressed the role of fully coherent energy loss (FCEL) in cold nuclear matter discussed throughout this Letter. FCEL is expected in all processes where the underlying partonic process, when viewed in the target nucleus rest frame, consists in forward scattering of an incoming energetic parton to an outgoing color charge [11,12] or a colorful system of partons [13]. It arises from induced gluon radiation with formation time $t_{f}$ much larger than the medium length, $t_{f} \gg L$, and associated to an energy spectrum $\omega d I / d \omega$ scaling in $x \equiv \omega / E$, with $E$ and $\omega$ the energies of the incoming parton and of the induced radiation, respectively. As a result, the average parton energy loss scales in $E, \Delta E_{\mathrm{FCEL}} \propto E$ [11], thus overwhelming the LPM energy loss $\Delta E_{\mathrm{LPM}} \propto L^{2}$ [14-17]. FCEL is predicted from first principles in various formalisms [11-13,18-22], including the saturation formalism $[21,22]$, and has been shown to be a key effect in quarkonium nuclear suppression in $p$-A collisions $[19,23,24]$. It is thus natural to investigate the effect of FCEL in other processes, such as open heavy flavor, light

Published by the American Physical Society under the terms of the Creative Commons Attribution 4.0 International license. Further distribution of this work must maintain attribution to the author(s) and the published article's title, journal citation, and DOI. Funded by SCOAP ${ }^{3}$. hadron, or jet production in $p$-A collisions. In the present study we focus on single inclusive light hadron production.

Our primary goal is to set a baseline for the quantitative role of FCEL in light hadron nuclear suppression, by taking into account only this effect. We show that similarly to quarkonium production, the effect of FCEL on light hadron production in $p$-A collisions is sizable, yet with novel features that will be underlined. In particular, FCEL depends on the global color charge of the parton pair produced in the partonic subprocess. As an interesting consequence, single hadron $p$-A production is sensitive to the color states [ $\mathrm{SU}\left(N_{c}\right)$ irreducible representations] of the parton pair, and thus to unusual color factors. Another goal of our study is to minimize the model dependence of FCEL baseline predictions. To that end, the $p p$ cross section is not taken from theory but determined from a fit to the data, and we thus predict the hadron nuclear modification factor $R_{p-\mathrm{A}}$ (due to FCEL), rather than absolute cross sections. As a result, our predictions of a significant hadron suppression arising from FCEL also have a remarkably small associated uncertainty.

These observations indicate that FCEL should be taken into account in phenomenological interpretations of the $p$-A data. It has been suggested to use present and future data on hadron production in $p$-A collisions $\left(h^{ \pm}[7,8], D / B\right.$ mesons [9,10], quarkonia [9]) as a reliable probe of NPDFs (and of saturation [4]), assuming other physical effects to be negligible. Our study shows that the latter assumption should be reconsidered, due to the presence of sizable FCEL effects. In particular, FCEL should be included in NPDF global fit analyses that use hadron production $p$-A data. In $p$-A collisions, electroweak processes where FCEL is absent [11] should be preferred for a direct extraction of NPDFs, as for instance weak boson [25] and Drell-Yan [26] production. Hadron production at the future EIC [27,28] would also be a direct probe of NPDFs and saturation since no FCEL is expected in deep inelastic scattering [11]. 


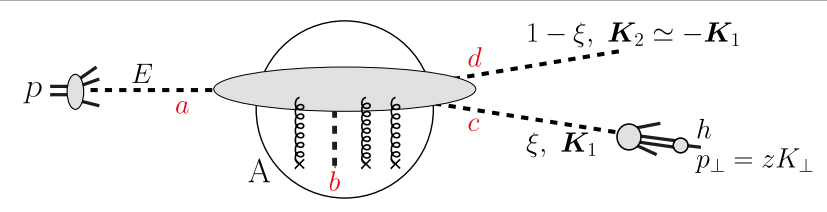

FIG. 1. Contribution to light hadron production in $p$-A collisions from the partonic process $a b \rightarrow c d$, as viewed in the nucleus rest frame, followed by the fragmentation $c \rightarrow h$ (represented here) or $d \rightarrow h$. In addition to the incoming parton $b$, the nucleus provides the nuclear broadening $\ell_{\perp A}$ arising from parton multiple scattering.

In order to implement FCEL in quarkonium hadroproduction [19,24], the differential production cross section in $p$-A is obtained from that in $p p$ collisions by rescaling the quarkonium energy by a factor $1+x$, where $x$ denotes the fractional energy loss, which is equivalent to a rapidity shift $\delta \equiv \ln (1+x)$,

$$
\frac{1}{A} \frac{d \sigma_{p-\mathrm{A}}^{\psi}(y)}{d y}=\int_{0}^{\delta_{\max }} d \delta \hat{\mathcal{P}}(x) \frac{d \sigma_{p p}^{\psi}(y+\delta)}{d y}
$$

In Eq. (1), $\delta_{\max }=\min \left(\ln 2, y_{\max }-y\right)$, with $y_{\max }$ the maximal quarkonium rapidity, and the quenching weight $\hat{\mathcal{P}}(x=$ $\left.e^{\delta}-1\right)$ is the fractional energy loss probability distribution associated to the production of a color octet compact $Q \bar{Q}$ pair $[19,24]$. The quenching weight is related to the induced coherent spectrum $d I / d x$ associated to the partonic process $g g \rightarrow G$ (with a final "massive gluon" $G$ ) derived and discussed in Refs. [11,12,19],

$$
\hat{\mathcal{P}}(x)=\frac{\partial}{\partial x} \exp \left\{-\left.\int_{x}^{\infty} d x^{\prime} \frac{d I}{d x^{\prime}}\right|_{g g \rightarrow G}\right\} .
$$

This approach has been successful in describing the quarkonium suppression reported in $p$-A collisions from the Super Proton Synchrotron to LHC energies $[19,24]$.

The procedure to implement FCEL in light hadron production in $p$-A collisions is analogous to quarkonium production, Eq. (1), despite new features. We will consider single inclusive hadron production at "large" $p_{\perp} \gg \ell_{\perp A}$, where $\ell_{\perp A}$ is the transverse momentum broadening of a fast gluon across the target $A$. In a leading-order picture, hadron production at $p_{\perp} \gg \ell_{\perp A}$ arises from $2 \rightarrow 2$ partonic processes $a b \rightarrow c d$, where $a$ and $b$ denote the partons taken in the incoming proton and target nucleus, respectively, and the partons of the final pair ("dijet" in the following) have transverse momenta $\boldsymbol{K}_{1}$ and $\boldsymbol{K}_{2} \simeq-\boldsymbol{K}_{1}$, with $K_{\perp} \equiv\left|\boldsymbol{K}_{1}\right| \gg \ell_{\perp A}$, and energy fractions $\xi$ and $1-\xi$ with respect to the energy $E$ of parton $a$ in the nucleus rest frame [29]. This is followed by the fragmentation of one parton of the final pair into the tagged hadron, which thus inherits the transverse momentum $p_{\perp}=z K_{\perp}$, where $z$ is the fragmentation variable. The process is illustrated in Fig. 1.
In general, calculating explicitly the induced coherent radiation spectrum associated to the production of a dijet (or multiparton system) may be complicated. When the induced radiation does not probe the dijet, however, the FCEL spectrum depends only on the dijet global color charge. In this "pointlike dijet approximation" (PDA), whose validity condition is $\ln \left(\ell_{\perp A}^{2} / x^{2} K_{\perp}^{2}\right) \gg 1[13,30]$ (ensuring in particular that, at its emission time, the induced radiation does not probe the dijet transverse size), the induced spectrum off a dijet in color state $R$ must coincide with that associated to $2 \rightarrow 1$ scattering $[11,12,19]$ applied to the case of an outgoing particle of Casimir $C_{R}$ and mass equal to the dijet mass $M_{\xi}=K_{\perp} / \sqrt{\xi(1-\xi)}$, namely [31],

$$
\left.x \frac{d I}{d x}\right|_{a b \rightarrow(c d)_{R}}=\left(C_{a}+C_{R}-C_{b}\right) \frac{\alpha_{s}}{\pi} \ln \left(\frac{1+\ell_{\perp A}^{2} / x^{2} M_{\xi}^{2}}{1+\ell_{\perp p}^{2} / x^{2} M_{\xi}^{2}}\right),
$$

where $C_{a}$ and $C_{b}$ are the Casimirs of partons $a$ and $b$. The scale $\ell_{\perp A}$ characterizing the FCEL spectrum (3) is given explicitly by $\ell_{\perp A}^{2}=\hat{q} L_{A}$, where $L_{A}$ is the average path length and $\hat{q}$ the transport coefficient in cold nuclear matter, parametrized as $\hat{q} \equiv \hat{q}_{0}\left(10^{-2} / x_{2}\right)^{0.3}$ at small $x_{2}$ [32].

In the PDA, implementing FCEL is simply achieved by separating the different dijet color states in the hadron production cross section, and performing the rapidity shift for each dijet color state separately [30]. Note that for a pointlike dijet, the same rapidity shift applies to the dijet, its constituent partons, and the tagged hadron. Two quantities are needed: (i) the probabilities $\rho_{R}$ for the dijet to be in color state $R$, which are fully determined from the $a b \rightarrow c d$ scattering amplitude (in the PDA the induced radiation responsible for FCEL does not probe the dijet and thus conserves its color state), and turn out to depend only on $\xi$ [30] and (ii) the FCEL quenching weight $\hat{\mathcal{P}}_{R}$ corresponding to a pointlike dijet in color state $R$, obtained by replacing in Eq. (2) the quantity $d I /\left.d x\right|_{g g \rightarrow G}$ by $d I /\left.d x\right|_{a b \rightarrow(c d)_{R}}$ given in (3).

The hadron production cross section in $p$-A collisions is thus written as [30]

$$
\begin{aligned}
\frac{1}{A} \frac{d \sigma_{p-\mathrm{A}}^{h}\left(y, p_{\perp}\right)}{d y d p_{\perp}}= & \sum_{R} \int_{0}^{\delta_{\max }} d \delta \int_{0}^{1} d \xi \\
& \times \rho_{R}(\xi) \hat{\mathcal{P}}_{R}\left(x, M_{\xi}\right) \frac{d \sigma_{p p}^{h}\left(y+\delta, p_{\perp}, \xi\right)}{d y d p_{\perp} d \xi}
\end{aligned}
$$

extending (1) to the case of light hadron production. In Eq. (4), the quenching weight is evaluated at $x=e^{\delta}-1$ and its dependence on $M_{\xi}$ is made explicit, as well as the dependence of the $p p$ and $p$-A cross sections on the hadron $p_{\perp}$. Let us emphasize that our implementation of FCEL 
effects consists in a different organization of the perturbative expansion as compared to next-to-leading order (NLO) approaches evaluating absolute $p$-A cross sections [34-39]. The latter studies in principle account for the induced radiation of a single gluon, as part of all NLO corrections. In our approach, the induced radiation is resummed to all orders through the expression of the quenching weight. This should be relevant when the spectrum (3) cannot be viewed as a genuine NLO correction, i.e., when $\ln \left(\ell_{\perp A}^{2} / x^{2} K_{\perp}^{2}\right) \gtrsim \mathcal{O}\left(1 / \alpha_{s}\right)$, consistently with the PDA used in our study.

Using (4), we obtain the nuclear production ratio in minimum bias $p$-A as compared to $p p$ collisions,

$$
\begin{aligned}
R_{p-\mathrm{A}}^{h}\left(y, p_{\perp}\right)= & \sum_{R} \int_{0}^{\delta_{\max }} d \delta\left\langle\rho_{R}(\xi) \hat{\mathcal{P}}_{R}\left(x, M_{\xi}\right)\right\rangle_{y+\delta, p_{\perp}} \\
& \times \frac{d \sigma_{p p}^{h}\left(y+\delta, p_{\perp}\right)}{d y d p_{\perp}} / \frac{d \sigma_{p p}^{h}\left(y, p_{\perp}\right)}{d y d p_{\perp}},
\end{aligned}
$$

where \langle\rangle$_{y, p_{\perp}}$ denotes the $\xi$ average in $p p$ dijet events where a hadron of rapidity $y$ and transverse momentum $p_{\perp}$ is produced [30].

In the spirit of the FCEL studies on quarkonium production $[19,24,40]$, the goal is to determine hadron suppression with a minimal model dependence. We use the following procedure, which introduces a limited set of parameters, the latter being then varied for a proper determination of theoretical uncertainties.

Defining the parameter $\bar{\xi}$ as the value which fulfils $\left\langle\sum_{R} \rho_{R}(\xi) \hat{\mathcal{P}}_{R}\left(x, M_{\xi}\right)\right\rangle_{y+\delta, p_{\perp}}=\sum_{R} \rho_{R}(\bar{\xi}) \hat{\mathcal{P}}_{R}\left(x, M_{\bar{\xi}}\right)$ (which existence is guaranteed by the mean value theorem), the nuclear production ratio (5) becomes

$$
\begin{gathered}
R_{p-\mathrm{A}}^{h}\left(y, p_{\perp}, \bar{\xi}\right)=\sum_{R} \rho_{R}(\bar{\xi}) R_{p-\mathrm{A}}^{R}\left(y, p_{\perp}, \bar{\xi}\right), \\
R_{p-\mathrm{A}}^{R}\left(y, p_{\perp}, \bar{\xi}\right)=\int_{0}^{\delta_{\max }} d \delta \hat{\mathcal{P}}_{R}\left(x, M_{\bar{\xi}}\right) \frac{\frac{d \sigma_{p p}^{h}\left(y+\delta, p_{\perp}\right)}{d y d p_{\perp}}}{\frac{d \sigma_{p p}^{h}\left(y, p_{\perp}\right)}{d y d p_{\perp}}} .
\end{gathered}
$$

Thus, $R_{p-\mathrm{A}}^{h}$ is the color average of the nuclear modification factors $R_{p \text {-A }}^{R}$ corresponding to a hadron produced from a dijet in color state $R$. The light hadron nuclear suppression due to FCEL is computed using Eqs. (6)-(7). The uncertainty associated to the choice of $\bar{\xi}$ will be estimated by taking $\bar{\xi}=0.50 \pm 0.25$, the default value $\bar{\xi}=0.50$ corresponding to the symmetric configuration of two jets of equal rapidity (which is most likely according to dihadron correlation measurements [41]), and the $\bar{\xi}$ variation range corresponding to a rapidity difference between the two jets of approximately $\Delta y= \pm \ln [\bar{\xi} /(1-\bar{\xi})] \simeq \pm 1$ unit.

The $p p$ cross section entering Eq. (7) is not taken from theory but from a fit to the data using a simple analytic form,

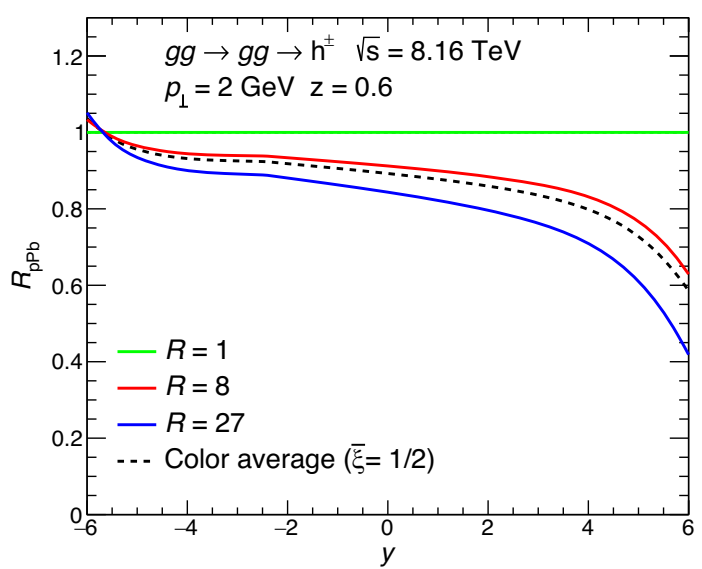

FIG. 2. Rapidity dependence of $R_{p \text {-A }}^{R}$, Eq. (7), at $p_{\perp}=2 \mathrm{GeV}$, in the $g g \rightarrow(g g)_{R}$ channel for $R=\mathbf{1}$ (green line), $R=\mathbf{8}$ (red line), and $R=27$ (blue line). The color-averaged nuclear modification factor $R_{p-\mathrm{A}}^{h}$, Eq. (6), is shown for $\bar{\xi}=1 / 2$ (dashed black line).

$d \sigma_{p p}^{h} / d p_{\perp} d y=f\left(p_{\perp}\right) \times\left[1-\left(2 p_{\perp} / \sqrt{s}\right) \cosh y\right]^{n}$ [24], where only the rapidity dependent factor, and thus the parameter $n$, is relevant when computing $R_{p-\mathrm{A}}^{h}$ from Eqs. (6)-(7). Using CMS double differential measurements in $p$ - $\mathrm{Pb}$ collisions at $\sqrt{s}=5.02 \mathrm{TeV}$ leads to the value $n=15 \pm 5$ [30]. The remaining parameters are the transport coefficient normalization $\hat{q}_{0}$, and the fragmentation variable $z$ (determining $K_{\perp}=p_{\perp} / z$ ), both entering only the expression of the spectrum (3). We use $\hat{q}_{0}=0.07 \pm 0.02 \mathrm{GeV}^{2} / \mathrm{fm}$, which is consistent with phenomenological estimates from a variety of processes (for instance radiative energy loss in $p$-A collisions [19] and in semi-inclusive DIS [42], or nuclear $p_{\perp}$ broadening in $p$-A collisions [43]), and take $z=0.6 \pm$ 0.2 based on NLO calculations of hadron production at the LHC [44,45]. Finally, as in Ref. [19], we set $\alpha_{s}=0.5$, $L_{p}=1.5 \mathrm{fm}$, and $L_{\mathrm{Pb}}=10.11 \mathrm{fm}$ (as determined from Glauber theory). In order to estimate the theoretical uncertainties, the quantities $n, \bar{\xi}, z$, and $\hat{q}_{0}$ are varied around their default values, which define the central prediction. Assuming the parameters to be uncorrelated, the uncertainty band of the model predictions is determined using the Hessian method [46] (applied in the context of FCEL effects on quarkonium production in [40]) from the variation of each parameter around its default value while keeping the other parameters fixed.

Calculations of light hadron suppression in $p-\mathrm{Pb}$ collisions at current top LHC energy, $\sqrt{s}=8.16 \mathrm{TeV}$, are shown here as a function of $p_{\perp}$ and $y$. For the sake of illustration, let us first consider the specific case of the $g g \rightarrow g g$ partonic process, and discuss the nuclear production ratio (7) for a final gluon pair in a given color state $R$, from which the "inclusive" hadron suppression can be obtained through the average (6) over color states. In Fig. 2 we show the rapidity dependence of $R_{p-\mathrm{A}}^{R}$ at fixed 

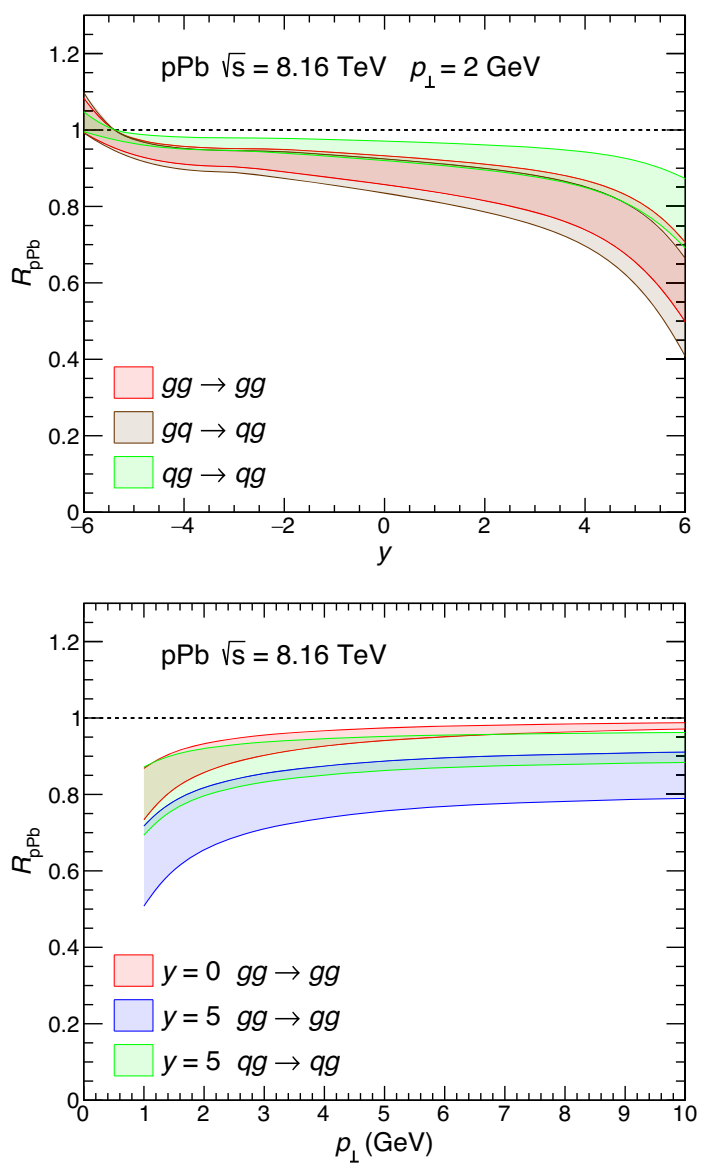

FIG. 3. FCEL effects on $R_{p-\mathrm{A}}^{h}$ for different channels, as a function of $y$ at $p_{\perp}=2 \mathrm{GeV}$ (top) and as a function of $p_{\perp}$ at $y=0$ and $y=5$ (bottom), in $p$-Pb collisions at $\sqrt{s}=8.16 \mathrm{TeV}$.

$p_{\perp}=2 \mathrm{GeV}$, for the color states $R=\mathbf{1}, \mathbf{8}, \mathbf{2 7}$ (having nonzero probabilities [30]), as well as the color average (dashed line). When the $g g$ final state is color singlet, the induced gluon spectrum (3) vanishes, and no FCEL effect is expected in this channel, $R_{p-\mathrm{A}}^{\mathbf{1}}=1$. More interesting is the suppression of the octet $g g$ final state, which shape is reminiscent of the suppression predicted for quarkonium [19]. The suppression gets quite strong at large $y, R_{p-\mathrm{A}}^{\mathbf{8}} \simeq 0.6$ at $y=6$, due to the steeply falling $p p$ cross section. Note that the steeply rising $p p$ cross section at very backward rapidity leads to a slight enhancement below $y \simeq-6$. For the 27-plet $g g$ final state, the suppression expected from FCEL follows the same pattern, but is more pronounced due to the larger Casimir, $C_{27}=2\left(N_{c}+1\right)$, in the prefactor of the induced gluon spectrum (3).

Light hadron suppression, obtained after averaging over the dijet color states, is displayed with its uncertainty band in Fig. 3, for the $g g \rightarrow g g$ channel, as a function of $y$ for $p_{\perp}=2 \mathrm{GeV}$ (Fig. 3, top) and as a function of $p_{\perp}$ for $y=0$ or $y=5$ (Fig. 3, bottom). In addition, results are shown for the $g q \rightarrow q g$ and $q g \rightarrow q g$ channels [which differ due to the opposite sign of $C_{a}-C_{b}$ in (3)]. Results for other channels

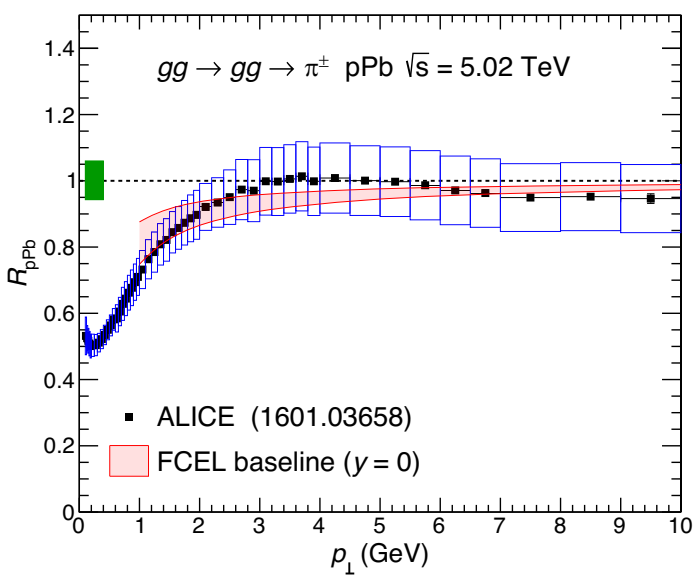

FIG. 4. FCEL effects on $R_{p-\mathrm{A}}^{h}$ as a function of $p_{\perp}$ in $p-\mathrm{Pb}$ collisions at $\sqrt{s}=5.02 \mathrm{TeV}$ (red band) in comparison to ALICE charged pion data [47].

turn out to be qualitatively similar and can be found in [30]. Because of the specific dependence of the induced gluon spectrum (3) in $M_{\xi} \propto K_{\perp}=p_{\perp} / z$, FCEL effects weaken with increasing $p_{\perp}$. At large positive rapidity, inclusive hadron production is dominated by the $q g \rightarrow q g$ scattering process [44]. Interestingly, the suppression expected in this process is less pronounced than in the $g g \rightarrow g g$ channel, due to the large negative contribution $C_{a}-C_{b}=C_{F}-N_{c}$ in the prefactor of Eq. (3) compared to $C_{a}-C_{b}=0$ in the $g g \rightarrow g g$ channel. Consequently, the resulting rapidity dependence of hadron suppression should be less steep than observed in the $g g \rightarrow g g$ channel only, because of the weighted sum over all partonic subprocesses.

We stress that the smallness of FCEL relative uncertainties (only $\sim 5 \%$ at midrapidity) is somehow expected within our approach: FCEL is a medium-induced effect (depending on the difference between coherent radiation spectra in $p$-A and $p p$ collisions), and is thus fully determined within perturbative QCD. Moreover, the parameters $\bar{\xi}, z$, and $\hat{q}_{0}$ enter the induced gluon spectrum (3) through a (large) logarithm, and their variation thus leads to small relative deviations around the central predictions. As for the parameter $n$, its variation affects negligibly the predictions, except at very large $|y|$ where it contributes to about half of the total relative uncertainty [30], which however remains moderate (see Fig. 3).

Let us now compare the FCEL expectations to the measurements of charged pion suppression in $p-\mathrm{Pb}$ collisions at $\sqrt{s}=5.02 \mathrm{TeV}$, performed at midrapidity by the ALICE experiment [47]. Calculations are carried out for the $g g \rightarrow g g$ channel which dominates hadron production around midrapidity. We have checked that including the $q g \rightarrow q g$ and $g q \rightarrow q g$ channels with proper weights according to NLO calculations [44] affects this prediction by less than $1 \%$. As shown in Fig. 4, good agreement is observed between data and FCEL baseline predictions. Therefore, the data sometimes attributed to saturation [3-6], NPDF 
effects [7], and the Cronin effect with initial-state energy loss [48], are as well consistent with fully coherent energy loss alone [49]. Since the uncertainties of the FCEL baseline prediction are significantly smaller than those of the measurements, taking into account FCEL should therefore provide strict constraints on other physical processes. It will be interesting to compare FCEL baseline predictions with future measurements by the $\mathrm{LHCb}$ experiment in the rapidity region $2<y<4.5$ [50]. In particular, the $g g \rightarrow q \bar{q}$ channel [30] will be generalized in a future study to the production of massive quarks, $g g \rightarrow Q \bar{Q}$, allowing for the calculation of FCEL effects on open heavy flavor production, recently measured by $\mathrm{LHCb}[51,52]$.

In this Letter, FCEL effects on light hadron production in $p$ - $\mathrm{Pb}$ collisions at LHC energies are computed for the first time, for different partonic subprocesses, and compared to ALICE data at midrapidity. The FCEL effects prove to be of the same order of magnitude as NPDF [7] or saturation [3-6] effects at midrapidity, and moreover subject to quite small uncertainties. This underlines the need to take into account FCEL in phenomenological studies of hadron production in $p$-A collisions. This study shows that FCEL effects are qualitatively similar for any given $2 \rightarrow 2$ partonic channel, and should pave the way for the systematic computation of FCEL effects in a (leading-order) perturbative QCD calculation of hadron production in $p$-A collisions. This program could be achieved at NLO using the FCEL spectrum associated to $1 \rightarrow 3$ forward scattering [13], eventually allowing for NPDF NLO global fit analyses taking FCEL effects into account.

This work is funded by "Agence Nationale de la Recherche," Grant No. ANR-COLDLOSS (Grant No. ANR-18-CE31-0024-02).

[1] F. Gelis, E. Iancu, J. Jalilian-Marian, and R. Venugopalan, Annu. Rev. Nucl. Part. Sci. 60, 463 (2010).

[2] N. Armesto, J. Phys. G 32, R367 (2006).

[3] P. Tribedy and R. Venugopalan, Phys. Lett. B 710, 125 (2012); 718, 1154(E) (2013).

[4] J. L. Albacete, A. Dumitru, H. Fujii, and Y. Nara, Nucl. Phys. A897, 1 (2013).

[5] A. H. Rezaeian, Phys. Lett. B 718, 1058 (2013).

[6] T. Lappi and H. Mäntysaari, Phys. Rev. D 88, 114020 (2013).

[7] I. Helenius, K. J. Eskola, H. Honkanen, and C. A. Salgado, J. High Energy Phys. 07 (2012) 073.

[8] P. Quiroga-Arias, J. G. Milhano, and U. A. Wiedemann, Phys. Rev. C 82, 034903 (2010).

[9] A. Kusina, J.-P. Lansberg, I. Schienbein, and H.-S. Shao, Phys. Rev. Lett. 121, 052004 (2018).

[10] K. J. Eskola, I. Helenius, P. Paakkinen, and H. Paukkunen, J. High Energy Phys. 05 (2020) 037.

[11] F. Arleo, S. Peigné, and T. Sami, Phys. Rev. D 83, 114036 (2011).
[12] F. Arleo, R. Kolevatov, and S. Peigné, Phys. Rev. D 93, 014006 (2016).

[13] S. Peigné and R. Kolevatov, J. High Energy Phys. 01 (2015) 141.

[14] R. Baier, Y. L. Dokshitzer, A. H. Mueller, S. Peigné, and D. Schiff, Nucl. Phys. B484, 265 (1997).

[15] R. Baier, Y. L. Dokshitzer, A. H. Mueller, S. Peigné, and D. Schiff, Nucl. Phys. B483, 291 (1997).

[16] B. G. Zakharov, JETP Lett. 63, 952 (1996).

[17] B. G. Zakharov, JETP Lett. 65, 615 (1997).

[18] N. Armesto, H. Ma, M. Martinez, Y. Mehtar-Tani, and C. A. Salgado, Phys. Lett. B 717, 280 (2012).

[19] F. Arleo and S. Peigné, J. High Energy Phys. 03 (2013) 122.

[20] N. Armesto, H. Ma, M. Martinez, Y. Mehtar-Tani, and C. A. Salgado, J. High Energy Phys. 12 (2013) 052.

[21] T. Liou and A. H. Mueller, Phys. Rev. D 89, 074026 (2014).

[22] S. Munier, S. Peigné, and E. Petreska, Phys. Rev. D 95, 014014 (2017).

[23] F. Arleo and S. Peigné, Phys. Rev. Lett. 109, 122301 (2012).

[24] F. Arleo, R. Kolevatov, S. Peigné, and M. Rustamova, J. High Energy Phys. 05 (2013) 155.

[25] H. Paukkunen and C. A. Salgado, J. High Energy Phys. 03 (2011) 071.

[26] F. Arleo and S. Peigné, Phys. Rev. D 95, 011502(R) (2017).

[27] A. Accardi et al., Eur. Phys. J. A 52, 268 (2016).

[28] U.S. Department of Energy Selects Brookhaven National Laboratory to Host Major New Nuclear Physics Facility, https://www.energy.gov/articles/us-department-energyselects-brookhaven-national-laboratory-host-major-newnuclear-physics.

[29] Note that in the terminology of Refs. [12,13,21,22], the processes $a b \rightarrow c d$ are nicknamed $a \rightarrow c d$ forward scatterings, emphasizing the energy flow of the process viewed in the nucleus rest frame (the incoming parton $b$ from the nucleus $A$ being softish in this frame).

[30] F. Arleo, F. Cougoulic, and S. Peigné, arXiv:2003.06337.

[31] We verified that the typical values of $x$ in our calculation satisfy $\ln \left(\ell_{\perp A}^{2} / x^{2} K_{\perp}^{2}\right) \sim 3-4$, thus justifying a posteriori the PDA and the use of Eq. (3).

[32] This follows from the proportionality of $\hat{q}$ to the gluon distribution $G(x)$ in a target nucleon, $\hat{q} \propto x G(x)$ [14], where $x$ should be taken as $x=\min \left(x_{0}, x_{2}\right)$ [19] (with $x_{0}=$ $1 /\left(2 m_{p} L_{A}\right)$ and $x_{2} \sim\left(2 K_{\perp} / \sqrt{s}\right) e^{-y}$ the longitudinal momentum fraction of the target parton in the $2 \rightarrow 2$ partonic subprocess), and from the parametrization $x G(x) \sim x^{\alpha}$, with $\alpha \simeq-0.3$, suggested by small- $x$ fits to HERA data [33]. We have checked that varying the exponent $\alpha$ by $\pm 10 \%$ would affect our results by less than $\pm 1 \%$ at $y=0$ and less than $\pm 5 \%$ at $y=6$.

[33] K. J. Golec-Biernat and M. Wüsthoff, Phys. Rev. D 59, 014017 (1998).

[34] G. A. Chirilli, B.-W. Xiao, and F. Yuan, Phys. Rev. Lett. 108, 122301 (2012).

[35] Z.-B. Kang, I. Vitev, and H. Xing, Phys. Rev. Lett. 113, 062002 (2014).

[36] B. Ducloué, T. Lappi, and Y. Zhu, Phys. Rev. D 93, 114016 (2016).

[37] H. Fujii and K. Watanabe, Nucl. Phys. A915, 1 (2013).

[38] B. Ducloué, T. Lappi, and H. Mäntysaari, Phys. Rev. D 91, 114005 (2015). 
[39] Y.-Q. Ma, R. Venugopalan, and H.-F. Zhang, Phys. Rev. D 92, 071901(R) (2015).

[40] F. Arleo and S. Peigné, J. High Energy Phys. 10 (2014) 73.

[41] V. Khachatryan et al. (CMS Collaboration), Phys. Lett. B 765, 193 (2017).

[42] W. K. Brooks and J. A. López, arXiv:2004.07236.

[43] F. Arleo and C.-J. Naïm, arXiv:2004.07188.

[44] R. Sassot, P. Zurita, and M. Stratmann, Phys. Rev. D 82, 074011 (2010).

[45] D. d'Enterria, K. J. Eskola, I. Helenius, and H. Paukkunen, Nucl. Phys. B883, 615 (2014).

[46] J. Pumplin, D. Stump, R. Brock, D. Casey, J. Huston, J. Kalk, H. L. Lai, and W. K. Tung, Phys. Rev. D 65, 014013 (2002).
[47] J. Adam et al. (ALICE Collaboration), Phys. Lett. B 760, 720 (2016).

[48] Z.-B. Kang, I. Vitev, and H. Xing, Phys. Lett. B 718, 482 (2012).

[49] Note however that the significant proton and antiproton enhancement measured by ALICE [47], reminiscent of the Cronin effect, is not consistent with the sole FCEL effects [30].

[50] R. Aaij et al. (LHCb Collaboration), Eur. Phys. J. C 74, 2888 (2014).

[51] R. Aaij et al. (LHCb Collaboration), Phys. Rev. D 99, 052011 (2019).

[52] R. Aaij et al. (LHCb Collaboration), Report No. CERNLHCb-CONF-2019-004, 2019. 